\title{
THE ASSOCIATION BETWEEN FRAILTY AND SHORT-TERM OUTCOMES IN AN INTENSIVE CARE UNIT REHABILITATION TRIAL: AN EXPLORATORY ANALYSIS
}

\author{
A. TAKAOKA ${ }^{1}$, D. HEELS-ANSDELL ${ }^{1}$, D.J. COOK ${ }^{1,2}$, M.E. KHO ${ }^{3}$ \\ 1. Department of Health Research Methods, Evidence, and Impact, Faculty of Health Sciences, McMaster University, Canada; 2. Department of Medicine, Faculty of Health Sciences, \\ McMaster University, Canada; 3. School of Rehabilitation Science, Faculty of Health Sciences, McMaster University, Physiotherapy Department, St. Joseph's Healthcare Hamilton, \\ Canada.

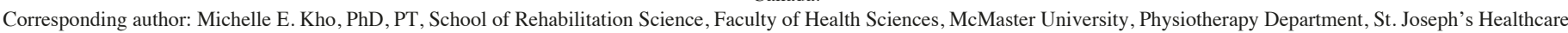 \\ Hamilton, Institute of Applied Health Sciences, 1400 Main St. W. Hamilton, ON L8S 1C7, Email: khome@mcmaster.ca, Telephone: (905) 525-9140 x28221, \\ Fax Number: (905) 524-0069
}

\begin{abstract}
Background: Physical therapy initiated early in an ICU stay may reduce functional deficits in critically ill patients; however, the association of frailty with outcomes in those receiving early in-ICU rehabilitation is unknown. Objective: To estimate the association between frailty and 3 outcomes in patients enrolled in an ICU randomized clinical trial (RCT). Design: Exploratory secondary analyses of the CYCLE pilot RCT (NCT02377830). Setting: 7 Canadian ICUs. Participants: Previously ambulatory critically ill adults. Intervention: Participants were randomized to early in-bed cycling plus routine physiotherapy versus early routine physiotherapy alone. Measurements: Using regression analyses, we modelled the association between pre-hospital Clinical Frailty Scale (CFS) scores, Physical Function in ICU Test-scored (PFIT-s), muscle strength, and mortality at hospital discharge, adjusting for illness severity (APACHE II) and the randomized intervention. We explored the influence of imputing mean PFIT-s and strength scores for decedents, and with listwise deletion of decedents in a sensitivity analysis. Results: Of 66 patients, 2 had missing data, 2 had incomplete data, and 21 died by hospital discharge. At hospital discharge for 66 patients, frailty was not associated with PFIT-s (mean difference $(\mathrm{MD})[95 \% \mathrm{CI}]=0.20,[-2.08,2.74])$ or muscle strength $(1.96,[-12.6,16.6])$. A sensitivity analysis yielded consistent results. Frailty was also not associated with hospital mortality (odds ratio 0.91, [0.28 to 2.93]). Conclusion: We found no association between pre-hospital frailty, physical function, strength, or mortality at hospital discharge in critically ill patients enrolled in an early rehabilitation trial. Larger sample sizes are needed to further explore the association of frailty with these outcomes at hospital discharge.
\end{abstract}

Key words: ICU, rehabilitation, frailty, outcomes, mechanical ventilation.

J Frailty Aging 2021;10(1)49-55

Published online October 5, 2020, http:/ / dx.doi.org/10.14283/jfa.2020.52

\section{Introduction}

Frailty is a patient health state characterized by losses in one or more domains of function $(1,2)$. In critically ill patients, a systematic review identified a 30\% (95\% CI: 29 to 32) baseline prevalence of frailty across 10 studies and 3030 participants (3). Regardless of frailty instrument used, patients with baseline frailty are consistently at a greater risk of functional dependence, disability, and mortality following critical illness (4-6). As the number of mechanically ventilated patients are projected to increase due to an aging baby boomer population (7), the impact of frailty is an urgent health concern across the continuum of care.

Rehabilitation initiated early in an ICU stay is a promising intervention to improve outcomes in critically ill adults (8). Increasing evidence has demonstrated that preserved physical fitness may be associated with lower 1-year mortality in elderly patients with frailty (9); however, to our knowledge, no studies have examined the association of frailty on the outcomes of patients receiving early rehabilitation in the ICU.

We recently completed a 7-centre pilot study of early leg cycle ergometry with mechanically ventilated patients who were ambulatory and independent prior to critical illness (10, 11). Using the study database, we conducted an exploratory analysis to evaluate the association between pre-hospital frailty status and hospital discharge measures of physical function, muscle strength, and mortality. We hypothesized that patients with frailty would have worse physical function, less muscle strength, and higher mortality at hospital discharge.

\section{Methods}

\section{Ethics}

This study was approved by the Hamilton Integrated Research Ethics Board (\#14-531).

\section{Design, Patients and Settings}

We conducted a preliminary, exploratory multivariable regression analyses of the CYCLE (Critical Care Cycling to Improve Lower Extremity Strength) pilot randomized controlled trial (RCT) (NCT02377830) that enrolled 66 critically ill patients across 7 Canadian ICUs. The methods 
and results of the RCT are described elsewhere $(10,11)$. Briefly, patients were included if they were $\geq 18$ years old, admitted within the first 4 days of mechanical ventilation and first 7 days of ICU, and independently ambulated with or without a gait aid before their critical illness. Primary exclusion criteria were any conditions impairing cycling, proven or suspected neuromuscular weakness, inability to follow commands in English, a temporary pacemaker, expected risk of hospital mortality $>90 \%$, palliative goals of care, or persistent exemptions precluding cycling. Enrolled patients were randomized to receive early in-bed cycle ergometry (30 minutes, 5 days/week, up to 28 days or ICU discharge) plus routine physiotherapy or early routine physiotherapy alone for the duration of their ICU stay.

\section{Dependent Variables}

At hospital discharge, trained physiotherapists blinded to treatment allocation measured function using the Physical Function in ICU Test-scored (PFIT-s) (12) and strength using the Medical Research Council Sum Score (MRC-SS) (13). Research coordinators documented hospital vital status (dead/ alive).

\section{Independent Variable}

Research coordinators evaluated frailty status in the 1-2 weeks before current hospital admission using the Clinical Frailty Scale (CFS) (2). These scores were generated at trial enrollment through family member and/or patient interviews and comprehensive chart reviews.

\section{Covariates}

We included covariates in our models to adjust for potential confounders. To address sample size limitations and to avoid overfitting models, we strategically limited the number of predictors in our models $(\geq 10$ participants per predictor in linear models; $\geq 10$ events per predictor in logistic models (14)). We purposefully selected 2 covariates a priori based on possible confounders of the relationships between pre-hospital frailty and our 3 outcomes. Our first covariate was illness severity (15-17) measured using the APACHE II (Acute Physiology and Chronic Health Evaluation II) score (18). We considered age as a covariate because of its association with both frailty and our outcomes of interest; however, since age contributes to overall APACHE II scores, we did not include it as a separate variable to avoid redundancy. Our second covariate was the randomized intervention, cycling plus routine physiotherapy versus routine physiotherapy alone, given the context of this analysis nested within the CYCLE pilot RCT.

Detailed descriptions of variables and covariates are provided in Table e1 (e-supplemental appendix).

\section{Analysis}

We tabulated descriptive statistics of baseline variables (e.g., age, sex, BMI, admission type, APACHE II scores (18),
Charlson Comorbidity Index (19), Functional Comorbidity Index (20), pre-ICU Functional Status Score for the ICU (FSS-ICU) (21), pre-ICU Katz Independence in Activities of Daily Living (Katz ADL) scores (22)) and trial-related characteristics (e.g., group allocation, time to first session, total days of rehabilitation, length of stay in ICU and hospital, outcomes) according to dichotomized frailty status, with frailty defined as a CFS score $\geq 5$. For continuous variables, we reported means and standard deviations (SD), or medians and interquartile ranges (IQR) if data were not normally distributed. We compared characteristics of patients with and without frailty using Student's t-tests or Mann-Whitney U tests as appropriate. We reported categorical variables as counts and proportions, and compared groups using Pearson's chi-square test.

We performed confirmatory multivariable linear regression to estimate the association between pre-hospital CFS scores, PFIT-s, and MRC-SS. We used binary logistic regression to model the association between pre-hospital CFS scores and hospital survival. In both models, we dichotomized patients by CFS scores for enhanced clinical interpretability. Linear regression results are presented as mean difference (MD) and 95\% confidence intervals (CI). Overall model statistics are reported as R2 and F values with degrees of freedom (df numerator, $\mathrm{df}$ denominator) in the e-supplemental appendix. Logistic regression odds ratios (OR) are presented with $95 \%$ confidence intervals. We considered a p-value $<0.05$ statistically significant for all tests. All analyses were performed using SPSS (IBM Corp. Released 2016. IBM SPSS Statistics for Macintosh, Version 25.0. Armonk, NY: IBM Corp.).

\section{Missing Data}

For patients with missing PFIT-s or MRC-SS data, when possible, we used ICU discharge scores under the rationale that ICU scores were based on the patient's own data and would provide a conservative estimate of outcome data at hospital discharge. For patients who died, we assigned PFIT-s and MRC-SS of 0 under the assumption that those who died would have little to no function or muscle strength. We conducted sensitivity analyses to explore the influence of these imputations for decedents (23).

\section{Sensitivity analyses}

Based on methodology adapted from Murphy et al. (23), we assessed our continuous outcome models with 1) listwise deletion, wherein only complete cases were included, and 2) imputed data using mean scores.

\section{Results}

We enrolled 66 patients in this pilot RCT (cycle intervention: $\mathrm{n}=36$, control: $\mathrm{n}=30$ ) with a mean (SD) age of $61.6(16.9)$ years and APACHE II score of 23.5 (8.6) (Table 1). The prevalence of frailty $(\mathrm{CFS} \geq 5)$ in our cohort was $26 \%$ (17/66) (Figure 1). Baseline characteristics were similar between those with and 


\section{THE JOURNAL OF FRAILTY \& AGING}

Table 1

Baseline characteristics of patients enrolled in the CYCLE pilot RCT, by frailty status

\begin{tabular}{|c|c|c|c|c|c|}
\hline Variable & $\begin{array}{c}\text { Overall } \\
(n=66)\end{array}$ & $\begin{array}{c}\text { Without Frailty }(\mathrm{CFS}<5) \\
(\mathrm{n}=49)\end{array}$ & $\begin{array}{c}\text { With Frailty }(\mathrm{CFS} \geq 5) \\
(\mathrm{n}=17)\end{array}$ & $\begin{array}{l}\text { Mean difference }^{\delta} \\
95 \% \text { CI }\end{array}$ & p-value \\
\hline Age in years Mean (SD) & $61.6(16.9)$ & $60.2(17.8)$ & $65.6(13.5)$ & $-5.4(-13.8,3.0)$ & $\mathrm{p}=0.20$ \\
\hline BMI Mean (SD) & $27.6(7.1)$ & $27.4(6.5)^{\gamma}$ & $28.1(8.8)$ & $-0.66(-5.5,4.2)$ & $\mathrm{p}=0.78$ \\
\hline Type & & & & & $\mathrm{p}=0.02^{\alpha}$ \\
\hline APACHE II Mean (SD) & $23.5(8.6)$ & $23.8(9.6)$ & $22.7(4.8)$ & $1.11(-2.5,4.7)$ & $\mathrm{p}=0.54$ \\
\hline Charlson Comorbidity Index, Mean (SD) & $1.9(1.6)$ & $1.9(1.7)$ & $2.1(1.5)$ & $0.26(-0.6,1.1)$ & $\mathrm{p}=0.55$ \\
\hline Functional Comorbidity Index, Median (IQR) & $2.0(0.0-2.0)$ & $1.0(0.0-3.5)$ & $2.0(1.0-4.0)$ & & $\mathrm{p}=0.36^{\beta}$ \\
\hline Pre-ICU admission FSS-ICU, Median (IQR) & $35.0(34.0-35.0)$ & $35.0(35.0-35.0)$ & $34.0(29.5-34.0)$ & & $\mathrm{p}<0.001^{\beta}$ \\
\hline
\end{tabular}

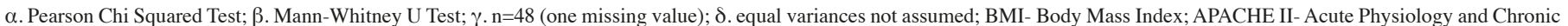
Health Index II Score; FSS-ICU- Functional status score for ICU; Katz ADL- Katz Activities of Daily living

Table 2

Trial and outcome characteristics of patients enrolled in the CYCLE pilot RCT, by frailty status

\begin{tabular}{|c|c|c|c|c|}
\hline Variable & Overall $(n=66)$ & $\begin{array}{l}\text { Without Frailty }(\mathrm{CFS}<5) \\
(\mathrm{n}=49)\end{array}$ & $\begin{array}{c}\text { With Frailty (CFS } \geq 5) \\
(n=17)\end{array}$ & p-value \\
\hline \multicolumn{5}{|l|}{ Group Allocation } \\
\hline Cycling plus routine PT, n (\%) & $36(54.5)$ & $28(57.1)$ & $8(47.1)$ & 0.47 \\
\hline Time from ICU admission to first session, median (IQR) & $3.0(2.0-4.0)$ & $3.0(2.0-4.0)$ & $2.0(2.0-4.0)$ & 0.92 \\
\hline Days of rehabilitation median (IQR) & $5.0(3.0-10.0)$ & $5.0(3.0-10.0)$ & $6.0(3.5-10.5)$ & 0.51 \\
\hline \multicolumn{5}{|l|}{ Length of stay, median (IQR) } \\
\hline ICU & $11.0(8.0-25.0)$ & $11.0(7.0-25.5)$ & $12.0(8.0-21.5)$ & 0.91 \\
\hline Hospital & $25.5(14.8-46.5)$ & $24.0(14.0-45.5)$ & $29.0(24.5-53.0)$ & 0.16 \\
\hline PFIT-s scores of survivors at hospital discharge ${ }^{\alpha}$ & $n=43$ & $\mathrm{n}=32$ & $\mathrm{n}=11$ & \\
\hline median (IQR) & $8.8(6.4-8.8)$ & $8.8(6.4-9.7)$ & $8.8(7.1-8.8)$ & \\
\hline mean $(\mathrm{SD})$ & $8.0(1.8)$ & $7.9(1.8)$ & $8.0(1.7)$ & \\
\hline MRC-SS ${ }^{\alpha . \beta}$ of survivors at hospital discharge & $n=41$ & $\mathrm{n}=31$ & $\mathrm{n}=10$ & \\
\hline median (IQR) & $55.0(50.0-58.0)$ & $55.0(50.0-58.0)$ & $55.0(51.5-58.3)$ & \\
\hline mean $(\mathrm{SD})$ & $53.6(5.3)$ & $53.4(5.1)$ & $54.1(5.9)$ & \\
\hline Mortality & $n=66$ & $n=49$ & $\mathrm{n}=17$ & \\
\hline $\mathrm{ICU}, \mathrm{n}(\%)$ & $18(27.3)$ & $14(28.6)$ & $4(23.5)$ & \\
\hline Hospital, n $(\%)$ & $22(33.3)$ & $16(32.7)$ & $6(35.3)$ & \\
\hline
\end{tabular}


without frailty, with the exception of more surgical admissions $(p=0.019)$ and unexpectedly higher Katz ADL scores $(p<0.001)$, and higher FSS-ICU $(\mathrm{p}<0.001)$ in those with frailty (Table 1). Twenty-two (33\%) patients died in hospital (36\% with frailty, $33 \%$ without frailty) (Table 2). There were no differences in trial-related physiotherapy characteristics, including time to first physiotherapy session or total days of rehabilitation, between those with frailty and those without (Table 2).

Figure 1

Clinical Frailty Scale (CFS) scores

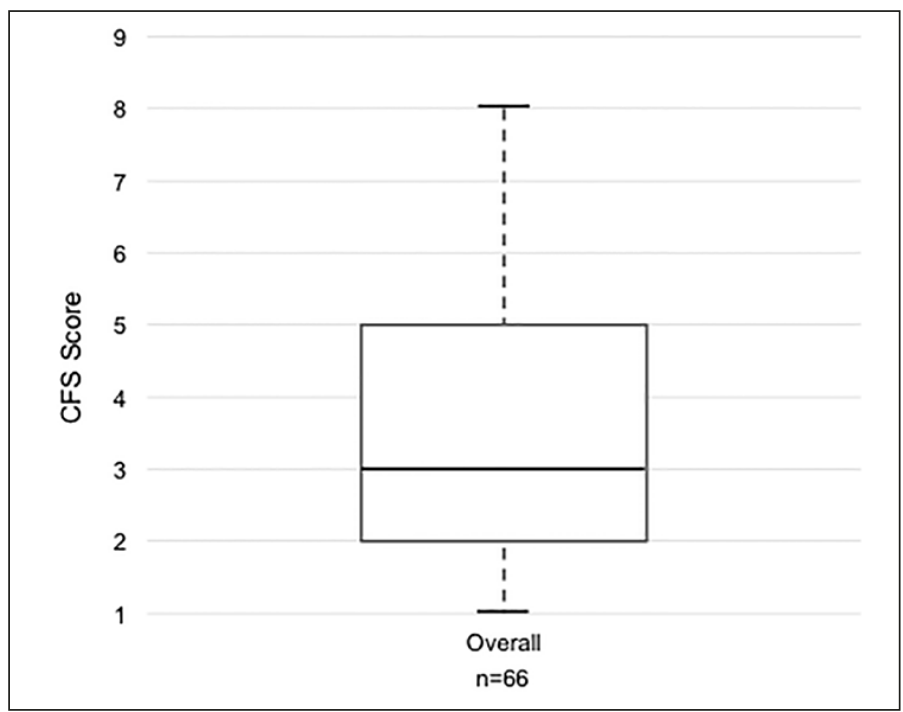

Distribution of Clinical Frailty Scale (CFS) scores. Overall prevalence of frailty (CFS $\geq 5)$ was $26 \%(17 / 66)$.
One patient completed PFIT-s and MRC-SS assessments while waiting to be discharged from hospital, but subsequently deteriorated, was re-admitted to ICU and died during the index hospitalization. The remaining 21 decedents were assigned PFIT-s and MRC-SS of 0. Four patients survived, but had some missing data. For the $2(3 \%)$ patients with missing PFIT-s and MRC-SS due to unexpected hospital discharge, and $2(3 \%)$ patients with partially completed MRC-SS (Figure 2), we used the corresponding ICU discharge measures in place of hospital discharge scores.

At hospital discharge, frailty was not associated with PFIT-s scores $(\mathrm{MD}=0.20,95 \% \mathrm{CI}$ : -2.08 to 2.74$)$ or muscle strength ( $\mathrm{MD}=1.96,95 \% \mathrm{CI}$ : -12.6 to 16.6$)$. These results were consistent in the sensitivity analyses. Frailty was not associated with in-hospital mortality $(\mathrm{OR}=0.91,95 \% \mathrm{CI}$ : 0.28 to 2.93$)$. We report full details of each model in eTable 2 and eTable 3, and results of the sensitivity analyses in eTable 4, and eFigures 1 and 2 in the e-supplemental appendix.

\section{Discussion}

In this cohort of previously ambulatory critically ill patients enrolled in a trial of early rehabilitation, our exploratory analyses demonstrated that pre-hospital frailty status measured using the CFS was not associated with physical function, muscle strength, nor mortality at hospital discharge, after adjusting for severity of illness and randomized assignment.

Our baseline frailty prevalence was 26\% (95\% CI: 15.4 to 36.6 ), which was similar to the $30 \%$ (95\% CI: 29 to 32) prevalence reported in previous prospective ICU studies

Figure 2

Flow diagram of patients enrolled in CYCLE Pilot RCT by frailty status

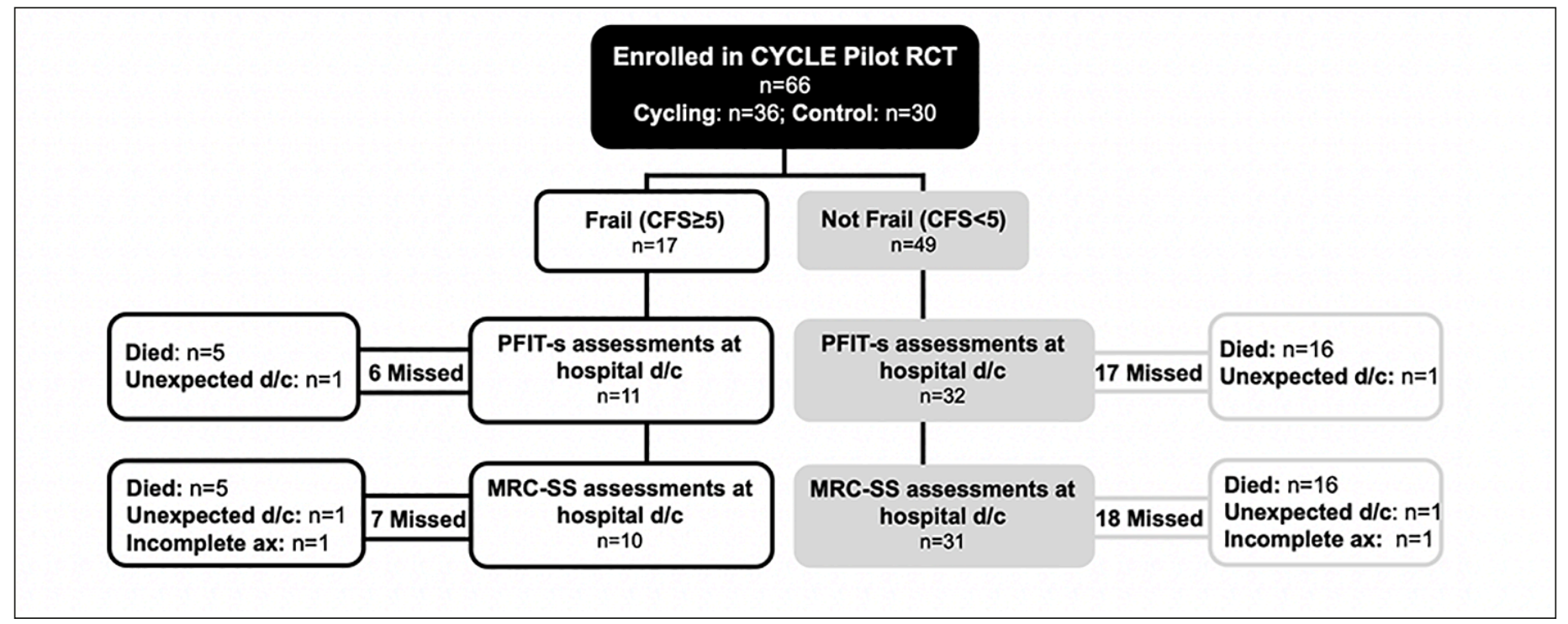

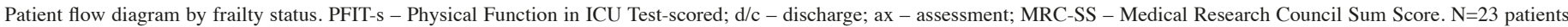

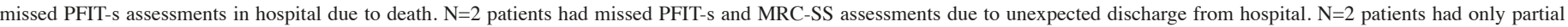
MRC-SS scores completed. 


\section{THE JOURNAL OF FRAILTY \& AGING}

summarized in a systematic review (3). Although the wide confidence interval surrounding our estimate indicates a high degree of imprecision, our slightly lower observed prevalence may reflect our inclusion criteria which required patients to ambulate independently before their critical illness $(10,11)$. The high level of baseline independence in this cohort may also explain the unexpectedly higher Katz ADL and FSS-ICU scores in those who were frail; however, these differences may also be due to chance, given our small sample size. Our results may also differ from this systematic review because the pooled estimate in the review included several distinct measures of frailty, including the CFS, Frailty Index (24), and Frailty Phenotype (1). Both the Frailty Index and Frailty Phenotype tend to report a higher frailty prevalence compared to the CFS $(25,26)$.

We found no association between frailty measured using the CFS and hospital mortality in our small cohort of patients. Our results are similar to 3 studies in critically ill patients that did not find an association between frailty and mortality at hospital discharge $(5,27,28)$. In contrast, 3 prospective studies demonstrated associations between higher CFS scores and hospital mortality $(4,25,26)$. Bagshaw et al. conducted a 6-center prospective cohort study enrolling 421 medicalsurgical patients with a frailty prevalence of $32.8 \%$ and demonstrated higher in-hospital mortality among patients with frailty (adjusted OR 1.81, 95\% CI: 1.09 to 3.01) (4). Of the remaining two studies, patients with frailty were also more likely to die in hospital $(25,26)$. Compared to our cohort, differences in previous study results could be due to patient population (high proportion of trauma patients), or use of unadjusted analyses (univariate logistic regression and Chi square) $(25,26)$.

Our results also differ from previous studies examining the relationship between frailty and function in ICU survivors. Three studies reported different results over time for the association between frailty and function $(4,5,28)$. Hope et al. reported an association between pre-ICU frailty disability in activities of daily living (ADLs) at 6-months after hospital discharge, but not at the time of hospital discharge (28). Brummel et al. demonstrated an association between higher CFS scores and greater odds of disability in instrumental activities of daily living (iADL), but not ADLs at 3- and 12- months post-hospital discharge (5). Bagshaw et al. demonstrated an independent association between pre-ICU frailty (CFS $\geq 5$ ) and the odds of self-reported new functional dependence at 6- and 12-months after hospital discharge (OR $2.25,95 \%$ CI 1.03 to 4.89 ) (4). Our results may differ from previous research because of different measurement methods (patient self-report vs. performance-based measures), timing of measurements, or the possibility of type-II error due to small sample size.

The previously cited studies did not document receipt of rehabilitation during the ICU stay. Our physical function results are similar to a single-centre retrospective study of 264 patients who received early progressive mobilization in a cardiovascular ICU (CVICU) (29). Patients $\geq 60$ years old, admitted to a 12-bed CVICU and meeting eligibility criteria, received early mobilization activities. Mobilization activities varied from bed/ cardiac chair (Level 1) to independent/modified independent walking $>50$ feet (Level 4). The prevalence of frailty measured by the CFS was $34.1 \%$ (90/264). In a multivariable model, after adjusting for age, sex, and severity of illness (APACHE III score), there was no difference in change in level of function at CVICU discharge between patients with or without frailty. Similar to other studies, patients with frailty had higher hospital mortality $(8.9 \%)$ than those without $(5.7 \%)$, however the authors did not conduct an adjusted analysis (29).

Differences in patient population, analysis methods, outcome measurement, exposure to ICU rehabilitation interventions, and study design may account for discordant results between the current study and previous research. Prospective and historical cohort studies may be limited by confounding as well as availability and quality of data. Previous studies had broad inclusion criteria, whereas our study focused on patients who could ambulate before their critical illness. Few studies documented receipt of ICU rehabilitation interventions. Our study included a sample of medical-surgical critically ill patients from 7 institutions, both the intervention group and control group started rehabilitation within a median (IQR) of 3 (2-4) days from ICU admission, and patients completed performance-based measures $(10,11)$. Rehabilitation in ICU is a promising intervention to improve muscle strength, functional capacity, and walking distance at ICU discharge. It may also shorten length of stay in both ICU and hospital, and improve health related quality of life at hospital discharge (30) and 6-months post discharge (31-33). We hypothesize that rehabilitation interventions could have a moderating effect on the functional deficits experienced by ICU survivors with frailty meeting strict inclusion criteria in clinical trials.

Our study had limitations. Our small sample size restricted the number of covariates that could be included in models and rendered our results underpowered and at risk of residual confounding. With a larger sample, we would have controlled for other known confounders including the functional comorbidity index, Katz-ADL, or body mass index. We dichotomized CFS scores for clinical interpretability. Furthermore, missing dependent variables due to death were imputed based on clinical rationale which may have created biased estimates (34); however, our sensitivity analyses explored the robustness of our imputation decisions.

Strengths of this study included the use of known confounders in regression models regardless of their statistical significance in the model (35). Trained physiotherapists, blinded to treatment allocation and frailty assessment conducted our performance-based function and strength measures. We had limited missing outcome data due to loss to follow up and managed these missing values using conservative estimates. Finally, this was the first prospective study of the association 
between frailty and outcomes of physical function, muscle strength, and mortality in a cohort of critically ill patients enrolled in an early ICU rehabilitation trial.

There is a projected future increase in our aging population and subsequently the number of mechanically ventilated patients (7). These findings support a larger research effort towards developing and studying interventions which aim to decrease healthcare system burden and resource utilization associated with the growing population of individuals living with frailty (36). To facilitate evaluation of the association of frailty with function, we suggest that future studies include common measures at similar time points. Recent papers on core outcome sets for studies of patients with acute respiratory failure (37), mechanical ventilation (38), and critical care rehabilitation studies (39) and frailty (in progress) support this premise.

\section{Conclusions}

We found no association between pre-hospital frailty and physical function, muscle strength, or mortality at hospital discharge in previously ambulatory critically ill patients enrolled in an early rehabilitation trial. Larger sample sizes are needed to further explore the influence of frailty on short-term outcomes after hospitalization.

\section{Declaration of Author(s) Competing Interests: None.}

Clinical Trials Registration Number: NCT02377830

Funding Statement: This work was supported by grants from Technology Evaluation in the Elderly Network Catalyst (now Canadian Frailty Network; CAT2014-05), Canadian Respiratory Research Network Emerging Research Leaders Initiative, Ontario Thoracic Society Grant-in-Aid and Canadian Institutes of Health Research Transitional Operating Grant (Award \#142327), Canada Foundation for Innovation, and the Ontario Ministry of Research and Innovation. MEK and DJC are each funded by a Canada Research Chair. AT was supported by Canadian Frailty Network Interdisciplinary Fellowship Award (IFP-2018). Restorative Therapies (Baltimore, MD) provided 2 RT-300 supine cycle ergometers for Toronto General Hospital and London Health Sciences sites for this research.

Acknowledgments: We would like to acknowledge the CYCLE Pilot RCT participating centers: St. Joseph's Healthcare Hamilton, Juravinski Hospital, Hamilton General Hospital, Toronto General Hospital, London Health Sciences - Victoria, St. Michael's Hospital, and Ottawa General Hospital. http://icucycle.com/cyclerct/

Open Access: This article is distributed under the terms of the Creative Commons Attribution 4.0 International License (http://creativecommons.org/licenses/by/4.0/), which permits use, duplication, adaptation, distribution and reproduction in any medium or format, as long as you give appropriate credit to the original author(s) and the source, provide a link to the Creative Commons license and indicate if changes were made.

\section{References}

1. Fried LP, Tangen CM, Walston J, Newman AB, Hirsch C, Gottdiener J, et al Frailty in Older Adults: Evidence for a Phenotype. J Gerontol Ser A. 2001 Mar 1;56(3):M146-57.

2. Rockwood K, Song X, MacKnight C, Bergman H, Hogan DB, McDowell I, et al. A global clinical measure of fitness and frailty in elderly people. CMAJ. 2005 Aug 30;173(5):489-95.

3. Muscedere J, Waters B, Varambally A, Bagshaw SM, Boyd JG, Maslove D, et al The impact of frailty on intensive care unit outcomes: a systematic review and metaanalysis. Intensive Care Med. 2017 Aug;43(8):1105-22.

4. Bagshaw SM, Stelfox HT, McDermid RC, Rolfson DB, Tsuyuki RT, Baig N, et al Association between frailty and short- and long-term outcomes among critically il patients: a multicentre prospective cohort study. CMAJ Can Med Assoc J J Assoc Medicale Can. 2014 Feb 4;186(2):E95-102.

5. Brummel NE, Bell SP, Girard TD, Pandharipande PP, Jackson JC, Morandi A, et al. Frailty and Subsequent Disability and Mortality among Patients with Critical Illness. Am J Respir Crit Care Med. 2017 01;196(1):64-72.

6. Ferrante LE, Pisani MA, Murphy TE, Gahbauer EA, Leo-Summers LS, Gill TM. The Association of Frailty With Post-ICU Disability, Nursing Home Admission, and Mortality: A Longitudinal Study. Chest. 2018;153(6):1378-86.

7. Needham DM, Bronskill SE, Calinawan JR, Sibbald WJ, Pronovost PJ, Laupacis A. Projected incidence of mechanical ventilation in Ontario to 2026: Preparing for the aging baby boomers. Crit Care Med. 2005 Mar;33(3):574-9.

8. Tipping CJ, Harrold M, Holland A, Romero L, Nisbet T, Hodgson CL. The effects of active mobilisation and rehabilitation in ICU on mortality and function: a systematic review. Intensive Care Med. 2017;43(2):171-83.

9. Åhlund K, Ekerstad N, Bäck M, Karlson BW, Öberg B. Preserved physical fitness is associated with lower 1-year mortality in frail elderly patients with a severe comorbidity burden. Clin Interv Aging. 2019;14:577-86.

10. Kho ME, Molloy AJ, Clarke F, Herridge MS, Koo KKY, Rudkowski J, et al. CYCLE pilot: a protocol for a pilot randomised study of early cycle ergometry versus routine physiotherapy in mechanically ventilated patients. BMJ Open. 2016 Apr 1;6(4):e011659.

11. Kho ME, Molloy AJ, Clarke FJ, Reid JC, Herridge MS, Karachi T, et al. Multicentre pilot randomised clinical trial of early in-bed cycle ergometry with ventilated patients. BMJ Open Respir Res. 2019;6(1):e000383.

12. Denehy L, de Morton NA, Skinner EH, Edbrooke L, Haines K, Warrillow S, et al. A physical function test for use in the intensive care unit: validity, responsiveness, and predictive utility of the physical function ICU test (scored). Phys Ther. 2013 Dec;93(12):1636-45.

13. Kleyweg RP, Meché FGAVD, Schmitz PIM. Interobserver agreement in the assessment of muscle strength and functional abilities in Guillain-Barré syndrome. Muscle Nerve. 1991;14(11):1103-9.

14. Harrell FE, Lee KL, Matchar DB, Reichert TA. Regression models for prognostic prediction: advantages, problems, and suggested solutions. Cancer Treat Rep. 1985 Oct;69(10):1071-7.

15. Fan E. Physical complications in survivors of acute lung injury: A 2-year longitudina prospective study. [Internet]. Johns Hopkins University; 2013. Available from: http:/ search.ebscohost.com/login. aspx?direct=true $\& \mathrm{db}=\operatorname{cin} 20 \& \mathrm{AN}=109863169 \&$ site $=$ eho st-live

16. Pfoh ER, Wozniak AW, Colantuoni E, Dinglas VD, Mendez-Tellez PA, Shanholtz C, et al. Physical declines occurring after hospital discharge in ARDS survivors: a 5-year longitudinal study. Intensive Care Med. 2016;42(10):1557-66.

17. Hodgson CL, Denehy L. Measuring physical function after ICU: one step at a time. Intensive Care Med. 2017;43(12):1901-3

18. Knaus WA, Draper EA, Wagner DP, Zimmerman JE. APACHE II: a severity of disease classification system. Crit Care Med. 1985 Oct;13(10):818-29.

19. Charlson ME, Pompei P, Ales KL, MacKenzie CR. A new method of classifying prognostic comorbidity in longitudinal studies: development and validation. J Chronic Dis. 1987;40(5):373-83.

20. Groll DL, To T, Bombardier C, Wright JG. The development of a comorbidity index with physical function as the outcome. J Clin Epidemiol. 2005 Jun;58(6):595-602.

21. Zanni JM, Korupolu R, Fan E, Pradhan P, Janjua K, Palmer JB, et al. Rehabilitation therapy and outcomes in acute respiratory failure: an observational pilot project. J Crit Care. 2010;25(2):254-62.

22. Katz S, Ford AB, Moskowitz RW, Jackson BA, Jaffe MW. Studies of Illness in the Aged. The index of ADL: A Standardized measure of Biological and Psychosocial Function. JAMA. 1963 Sep 21;185:914-9.

23. Murphy TE, Gill TM, Leo-Summers LS, Gahbauer EA, Pisani MA, Ferrante LE. The Competing Risk of Death in Longitudinal Geriatric Outcomes. J Am Geriatr Soc. 2019;67(2):357-62

24. Mitnitski A, Mogilner A, Rockwood K. Accumulation of deficits as a proxy measure of aging. Eur BMC. 2001;(1):232-336.

25. Le Maguet P, Roquilly A, Lasocki S, Asehnoune K, Carise E, Saint Martin M, et al Prevalence and impact of frailty on mortality in elderly ICU patients: a prospective, multicenter, observational study. Intensive Care Med. 2014 May;40(5):674-82. 


\section{THE JOURNAL OF FRAILTY \& AGING}

26. Tipping CJ, Hodgson CL, Harrold M, Chan T, Holland AE. Frailty in Patients With Trauma Who Are Critically Ill: A Prospective Observational Study to Determine Feasibility, Concordance, and Construct and Predictive Validity of 2 Frailty Measures. Phys Ther. 2019 01;99(8):1089-97.

27. Fisher C, Karalapillai DK, Bailey M, Glassford NG, Bellomo R, Jones D. Predicting intensive care and hospital outcome with the Dalhousie Clinical Frailty Scale: a pilot assessment. Anaesth Intensive Care. 2015 May;43(3):361-8.

28. Hope AA, Hsieh SJ, Petti A, Hurtado-Sbordoni M, Verghese J, Gong MN. Assessing the Usefulness and Validity of Frailty Markers in Critically Ill Adults. Ann Am Thorac Soc. 2017 Jun;14(6):952-9.

29. Goldfarb M, Afilalo J, Chan A, Herscovici R, Cercek B. Early mobility in frail and non-frail older adults admitted to the cardiovascular intensive care unit. J Crit Care. 2018;47:9-14

30. Burtin C; Clerckx B; Robbeets C; Ferdinande P; Langer D; Troosters T; Hermans G; Decramer M; Gosselink R. Early exercise in critically ill patients enhances short-term functional recovery. Crit Care Med 2009 Sep3792499-2505. 2009;

31. Kayambu G, Boots R, Paratz J. Early physical rehabilitation in intensive care patients with sepsis syndromes-a randomised controlled trial. Physiother U K. 2015;101:eS735-

32. Arias-Fernández P, Romero-Martin M, Gómez-Salgado J, Fernández-García D. Rehabilitation and early mobilization in the critical patient: systematic review. J Phys Ther Sci. 2018 Sep;30(9):1193-201.

33. Zang K, Chen B, Wang M, Chen D, Hui L, Guo S, et al. The effect of early mobilization in critically ill patients: A meta-analysis. Nurs Crit Care. 2019;(9808649, $\mathrm{c} 3 \mathrm{k})$.
34. Graham JW. Missing data analysis: making it work in the real world. Annu Rev Psychol. 2009;60:549-76.

35. Lederer DJ, Bell SC, Branson RD, Chalmers JD, Marshall R, Maslove DM, et al. Control of Confounding and Reporting of Results in Causal Inference Studies. Guidance for Authors from Editors of Respiratory, Sleep, and Critical Care Journals. Ann Am Thorac Soc. 2019;16(1):22-8.

36. Kokorelias KM, Munce S, McGilton KS, Cronin SM, Wang CD, Eftekhar P, et al. Frailty in the context of rehabilitation interventions for adults: protocol for a scoping review. BMJ Open. 2019 Feb 1;9(2):e024838.

37. Needham DM, Sepulveda KA, Dinglas VD, Chessare CM, Friedman LA, Bingham $\mathrm{CO}$, et al. Core Outcome Measures for Clinical Research in Acute Respiratory Failure Survivors. An International Modified Delphi Consensus Study. Am J Respir Crit Care Med. 2017 01;196(9):1122-30.

38. Blackwood B, Ringrow S, Clarke M, Marshall JC, Connolly B, Rose L, et al A Core Outcome Set for Critical Care Ventilation Trials. Crit Care Med. 2019 Oct; 47(10):1324-31.

39. Connolly B, Denehy L, Hart N, Pattison N, Williamson P, Blackwood B. Physical Rehabilitation Core Outcomes In Critical illness (PRACTICE): protocol for development of a core outcome set. Trials. 2018 May 25;19(1):294. 